\title{
Prognostic and Risk Factors in Patients with Locally Advanced Cutaneous Squamous Cell Carcinoma of the Trunk and Extremities
}

\author{
de Lima Vazquez Vinicius, ${ }^{1}$ Cristovam Scapulatempo, ${ }^{1}$ Natalia Martins Perpetuo, ${ }^{1}$ \\ Faheez Mohamed, ${ }^{2}$ Teóclito Sachetto de Carvalho, ${ }^{1}$ Antônio Talvane Torres de Oliveira, ${ }^{1}$ \\ José Getúlio Martins Segalla, ${ }^{3}$ and André Lopes Carvalho ${ }^{1}$ \\ ${ }^{1}$ Department of Surgery and Pathology, Hospital de Câncer de Barretos, Rua Antenor Duarte Villela, \\ 1331-Bairro Paulo Prata, 14784-400 Barretos, SP, Brazil \\ ${ }^{2}$ Division of Surgery, Basingstoke and North Hampshire NHS Foundation Trust, ldermaston Road Basingstoke, \\ Hampshire RG24 9NA, UK \\ ${ }^{3}$ Department of Clinical Oncology, Hospital Amaral Carvalho, R Dona Silveria, 150 17203-570 Jaú SP, Brazil
}

Correspondence should be addressed to de Lima Vazquez Vinicius, viniciusvazquez@gmail.com

Received 14 December 2010; Revised 23 January 2011; Accepted 16 March 2011

Academic Editor: Mohammed Kashani-Sabet

Copyright ( $) 2011$ de Lima Vazquez Vinicius et al. This is an open access article distributed under the Creative Commons Attribution License, which permits unrestricted use, distribution, and reproduction in any medium, provided the original work is properly cited.

55 patients with advanced cutaneous squamous cell carcinoma (CSCC) of the trunk and extremities were studied. A Tissue Microarray was constructed using immunohistochemistry to quantify expression of the HER family, E-cadherins, and podoplanin. Clinical and histopathological factors related to lymph node metastasis and prognosis were also established. Primary tumor positivity was $25.5 \%$ for EGFR, $87.3 \%$ for HER-3, and 48.1\% for HER-4. Metastases were positive for EGFR in 41.7\%, for HER-3 in 83.3\%, and HER-4 in 43.5\%. HER-2 was negative in all samples. Membrane E-cadherin and cytoplasmic E-cadherin were positive in $47.3 \%$ and $30.2 \%$ of primary tumors and $45.5 \%$ and $27.3 \%$ of metastases, respectively. Podoplanin was positive in $41.8 \%$ of primary tumors and $41.7 \%$ of metastases. Intratumoral lymphocytic infiltrate was associated with lymph node metastasis. Patients with T3 tumors had better cancer-specific survival (CSS) than those with T4 tumors; patients with no lymph node involvement had better CSS than patients with N1 tumors. Undifferentiated tumors and hyperexpression of podoplanin were negative prognostic indicators on multivariate analysis.

\section{Introduction}

"Locally advanced cutaneous squamous cell carcinoma of the trunk and extremities has a poor prognosis. This study identified prognostic factors including podoplanin, a novel molecular marker."

Cutaneous squamous cell carcinoma (CSCC) has a high incidence worldwide particularly in the sun exposed skin of Caucasians [1-5]. The majority of cases are readily treatable by simple excision or radiotherapy with a good chance of achieving cure. However, locally advanced tumors may present with local recurrence, lymph node or distant metastasis [6-12]. Unlike head and neck tumors, where the presence of lymph node metastases and disease progression are more common, Prognostic factors for advanced tumors of the trunk and extremities are not well established. Clinical and epidemiologic factors are poorly understood with only a few reports in the literature $[10,11,13-15]$. Knowledge of the role of molecular markers in tumor progression and metastasis is limited. The tyrosine kinases Human Epidermal Receptor (HER) family (Epidermal Growth Factor Receptor (EGFR), HER-2, HER-3, and HER-4) are transmembrane glycoproteins related to cell proliferation, differentiation, and apoptosis [16]. Altered expression of the HER family is associated with several epithelial tumors such as breast carcinoma 
and esophageal squamous cell carcinoma [17-20]. Small studies have also shown altered HER expression in localized squamous cell carcinoma when compared to normal skin [21-24]. HER expression in advanced CSCC of the trunk and extremities is not well studied and may be related to prognosis allowing the use of targeted therapies that block the HER pathway.

E-cadherin is a transmembrane glycoprotein, and it is a mediator of calcium-dependent cell-cell adhesion in normal cells [25]. Reduced cell-cell adhesiveness is considered important in both early and late carcinogenis [25, 26]. High E-cadherin expression in cell cytoplasm and low expression in the cell membrane are associated with tumor aggressiveness in different cancers, (i.e., lung cancer).

Podoplanin is a membrane protein found on lymphatic vessel endothelium. Its function is poorly understood although it may govern endothelial motility, and its absence in animal studies is associated with lymphedema and malformation of lymphatic vessels [27]. The aim of this study was to determine the expression of markers such as the HER family, E-cadherin, and Podoplanin in a consecutive series of locally advanced CSCC of the trunk and extremities and to define clinical, pathological, and molecular factors related to lymph node metastasis and survival.

\section{Methods}

A retrospective study of patients with locally advanced (American Joint Committee on Cancer staging T3 and T4 ) CSCC of the trunk and extremities admitted to two cancer institutions in Brazil (Barretos Cancer Hospital and Amaral Carvalho Hospital) between 1997 and 2006 was performed. Only those patients with tumor paraffin blocks available for analysis were included. Patients with tumor infiltration of the head and neck or genital area and those with a previous cancer diagnosis other than cutaneous basal cell carcinoma were excluded. This was to avoid difficulties in identifying origin of metastasis and cause of death. 55 consecutive patients admitted and treated from October 1997 to March 2006 with a pathologic diagnosis of squamous cell carcinoma were evaluated. Patients had to have stage T3 (tumor $>5 \mathrm{~cm}$ ) or T4 (invasive of deep extradermal structures) tumors according to the 2002 American Joint Committee on Cancer (AJCC) staging system. Institutional Review Board approval was obtained and all clinical information retrospectively collected from medical records.

2.1. Demographic and Clinical Characteristics. Demographic and clinical variables assessed included age, gender, ethnicity, previous chronic skin lesions (burns, scars, varicose ulcers and others) at the site of the tumor, patient residence (rural or urban), anatomic site, and treatment. Patterns of lymph node metastases, recurrence, and survival outcomes were also recorded.

Lymph node metastasis was classified as follow: N0: patients with no evidence of lymph node metastasis at presentation; N1: patients with lymph node metastasis at presentation. We considered lymph node metastasis at presentation (N1) or recurrence as the endpoint for risk of lymph node metastasis. The endpoint for survival was death from cancer. Only clinically involved lymph nodes were removed and no elective or sentinel node dissections were performed.

2.2. Pathology. 55 primary tumors and 22 lymph node metastases were available for pathological review by two pathologists. The pathological variables analyzed were number of mitosis $/ \mathrm{mm}^{2}$, deepest tumor diameter (Breslow depth), tumor grade I to III as previously described [28], perineural or perivascular infiltration, and intratumoral and peritumoral lymphocytic infiltration. Any lymphocytic infiltration was quantified as positive. Breslow depth was available in 44 cases.

2.3. Tissue Micro array. After pathological review, the most representative tumor area in the paraffin block was selected for creation of a tissue microarray (TMA). Both primary tumors and lymph node metastases were selected. A Manual Tissue Arrayer I, (Beecher Instruments, EUA) was used to obtain two cylinders of 1.0 millimeter in diameter from each paraffin bloc. These were implanted into the receptor paraffin block (TMA). Fifty slides were obtained and numbered from the TMA. For sample quality analysis slide numbers 1,25 , and 50 were stained with hematoxilin and eosin, and the most representative was chosen, and the other slides studied were subsequent to this.

2.4. Immunohistochemistry. Deparaffinization of the sections was done with xylene for 15 minutes at $60^{\circ} \mathrm{C}$, followed by 15 minutes at room temperature. The sections were then washed 3 times for 30 seconds with 100\%, 95\%, 80\%, and $70 \%$ ethanol before washing in water. Endogenous peroxidase was blocked by incubating the sections in $6 \%$ hydrogen peroxide in methanol. The sections were then washed with phosphate buffered saline (PBS) $10 \mathrm{mM} \mathrm{pH} 7.4$ for 5 minutes. Incubation followed, as described by Hsu and Raine [29] with the specific antibody diluted in PBS with $1.0 \%$ bovin serum albumin (Sigma USA) and $0.1 \% \mathrm{NaN}_{3}$ for 30 minutes at $37^{\circ} \mathrm{C}$ and for 16 hours at $4^{\circ} \mathrm{C}$.

For EGFR the H11 clone (DAKO) was used, diluted $1: 100$ in an autoclave with EDTA at $\mathrm{pH}$ 8.0. The following HER family polyclonal antigens were used all with citrate at pH 6.0: HER-2 (DAKO) diluted 11500 in a moist chamber, HER-3 (Neomarkers) $1: 100$ in an autoclave, (HER-4) (Neomarkers) $1: 300$ in an autoclave. The E-cadherin NCH38 (DAKO) monoclonal antigen was diluted $1: 600$ in a moist chamber with EDTA/TRIS at pH 9.0. The Podoplanin D2-40 clone (DAKO) was used at a dilution of $1: 200$ in a moist chamber with EDTA/TRIS at $\mathrm{pH}$ 9.0. After incubation they were washed with PBS 3 times for 5 minutes each and then antigen amplification was performed. After amplification they were again washed with PBS 3 times for 5 minutes each. Reactions were visualized with $0.6 \mathrm{mg} / \mathrm{mL} 3^{\prime}-3^{\prime}$ diaminobencidine tetrahydrochloride and $0.06 \%$ hydrogen peroxide in a $1 \%$ PBS solution for 5 minutes at $37^{\circ} \mathrm{C}$. The final reaction was a brown color deposit in the cell area where the antigen-antibody reaction had occurred. 
TABle 1: Demographic, clinical, therapeutic, and pathological characteristics of patients with locally advanced cutaneous squamous cell carcinoma of trunk and extremities.

\begin{tabular}{|c|c|c|c|}
\hline Characteristics & $n(\%)$ & Characteristics & $n(\%)$ \\
\hline Gender & & Lymph node metastasis location & \\
\hline Male & $32(58.2)$ & Axila & $15(60.0)$ \\
\hline Female & $23(41.8)$ & Groin & $10(40.0)$ \\
\hline Race & & Lymph node metastasis treatment & \\
\hline Caucasian & $49(89.1)$ & Linphadenectomy & $28(93.3)$ \\
\hline African & $6(10.9)$ & No treatment & $2(6.7)$ \\
\hline Residence & & Local or lymph node recurrence after lymphadenectomy & \\
\hline Rural & $9(16.4)$ & No & $13(46.4)$ \\
\hline \multirow[t]{2}{*}{ Urban } & $46(83.6)$ & Local & $8(28.6)$ \\
\hline & & Lymph node & $7(25.0)$ \\
\hline Chronic sun exposure & & Distant metastasis & \\
\hline Yes & $27(49.1)$ & No & $58(92.1)$ \\
\hline No & $12(21.8)$ & Cutaneous & $1(1.6)$ \\
\hline n.a. & $16(29.1)$ & Visceral & $4(6.3)$ \\
\hline Anatomical localization & & Lymph node metastasis treatment & \\
\hline Lower extremities & $22(40.0)$ & Linphadenectomy & $24(96.0)$ \\
\hline Upper extremities & $23(41.8)$ & No treatment & $1(4.0)$ \\
\hline Trunk & $10(18.2)$ & & \\
\hline Non-cancer previous lesion & & Tumor grade & \\
\hline Yes & $13(23.6)$ & I & $25(45.5)$ \\
\hline \multirow[t]{2}{*}{ No } & $42(76.4)$ & II & $27(49.0)$ \\
\hline & & III & $3(5.5)$ \\
\hline T classification & & Intratumoral lymphocitic infiltrate & \\
\hline $\mathrm{T} 3$ & $33(60.0)$ & Negative & $12(21.8)$ \\
\hline $\mathrm{T} 4$ & $22(40.0)$ & Positive & $43(78.2)$ \\
\hline $\mathrm{N}$ classification & & Peritumoral lymphocitic infiltrate & \\
\hline No & $41(74.5)$ & Negative & $9(16.4)$ \\
\hline N1 & $14(25.5)$ & Positive & $46(83.6)$ \\
\hline Clinical stage & & Vascular infiltrate & \\
\hline II & $27(49.1)$ & Negative & $48(87.3)$ \\
\hline III & $28(50.9)$ & Positive & $7(12.7)$ \\
\hline Treatment of primary tumor & & Perineural infiltrate & \\
\hline surgery & & Negative & $54(98.2)$ \\
\hline Local resection with primary closure & $1(1.9)$ & Positive & $1(1.8)$ \\
\hline Local resection with reconstruction & $18(32.7)$ & & \\
\hline Amputation/disarticulation & $21(38.2)$ & & \\
\hline Local resection with open wound & $5(9.1)$ & & \\
\hline Radiation therapy & $8(14.5)$ & & \\
\hline No treatment & $2(3.6)$ & & \\
\hline
\end{tabular}

n.a.: not available.

2.5. Immunohistochemistry Expressions. The EGFR, HER-3, and HER-4 expressions were evaluated semiquantitatively according to the method described by Lager et al. [30]. Areas with more intense reaction were selected, and the intensity of cytoplasm and/or membrane reaction was classified as 0 negative; + weak; ++ moderate; +++ intense. For the study, tumors classified as 0 or + were considered negative and tumors classified as ++ or +++ were considered positive.
HER-2 expression was evaluated semiquantitatively according to the intensity of reaction in the cytoplasmatic membrane. Negative or weak reaction in less than $10 \%$ of cells was classified as " 0 ", weak reaction in more than $10 \%$ as "+", moderate reaction in more than $10 \%$ as " ++ ", and strong reaction in more than $10 \%$ as " +++ " positivity. For the analysis, 0 and + were considered negative and ++ and +++ positive [31]. 
TABLE 2: Univariate analysis of risk factors for lymph node metastasis in patients with locally advanced cutaneous squamous cell carcinoma of trunk and extremities.

\begin{tabular}{|c|c|c|c|}
\hline Variables & + Lymph node metastasis $n(\%)$ & - Lymph node metastasis $n(\%)$ & $p$ \\
\hline \multicolumn{4}{|l|}{ Clinical } \\
\hline \multicolumn{4}{|l|}{ Gender } \\
\hline Male & $16(64.0 \%)$ & $16(53.3 \%)$ & \multirow{2}{*}{0.584} \\
\hline Female & $9(36.0 \%)$ & $14(46.7 \%)$ & \\
\hline \multicolumn{4}{|l|}{ Race } \\
\hline Caucasian & $22(88.0 \%)$ & $27(90.0 \%)$ & \multirow{2}{*}{1.000} \\
\hline African & $3(12.0 \%)$ & $3(10.0 \%)$ & \\
\hline \multicolumn{4}{|l|}{ Ambient } \\
\hline Rural & $4(16.0 \%)$ & $5(16.7 \%)$ & \multirow{2}{*}{1.000} \\
\hline Urban & $21(84.0 \%)$ & $25(83.3 \%)$ & \\
\hline \multicolumn{4}{|l|}{ Chronic Sun exposure } \\
\hline Yes & $14(66.7 \%)$ & $13(72.2 \%)$ & \multirow{2}{*}{0.742} \\
\hline No & $7(33.3 \%)$ & $5(27.8 \%)$ & \\
\hline \multicolumn{4}{|c|}{ Anatomic location of primary tumor } \\
\hline Lower extremities & $8(32.0 \%)$ & $14(46.7 \%)$ & \multirow{3}{*}{0.151} \\
\hline Upper extremities & $14(56.0 \%)$ & $9(30.0 \%)$ & \\
\hline Trunk & $3(12.0 \%)$ & $7(23.3 \%)$ & \\
\hline \multicolumn{4}{|c|}{ Previous non neoplasic lesion } \\
\hline Yes & $7(72.0 \%)$ & $6(20.0 \%)$ & \multirow{2}{*}{0.537} \\
\hline No & $18(28.0 \%)$ & $24(80.0 \%)$ & \\
\hline \multicolumn{4}{|l|}{ TNM Classification } \\
\hline T3 & $13(52.0 \%)$ & $20(66.7 \%)$ & \multirow{2}{*}{0.286} \\
\hline $\mathrm{T} 4$ & $12(48.0 \%)$ & $10(33.3 \%)$ & \\
\hline \multicolumn{4}{|l|}{ Tumor length* } \\
\hline Breslow 0-8 mm & $14(56.0 \%)$ & $8(44.5 \%)$ & \multirow{2}{*}{0.533} \\
\hline Breslow $>8 \mathrm{~mm}$ & $10(44.0 \%)$ & $10(55.5 \%)$ & \\
\hline \multicolumn{4}{|l|}{ Mitosis/mm² } \\
\hline $0-3$ & $22(44.9 \%)$ & $27(55.1 \%)$ & \multirow{2}{*}{0.573} \\
\hline$>3$ & $3(50.0 \%)$ & $3(50.0 \%)$ & \\
\hline \multicolumn{4}{|l|}{ Tumor grade } \\
\hline I & $11(44.0 \%)$ & $14(46.6 \%)$ & \multirow{2}{*}{1.000} \\
\hline II-III & $14(56.0 \%)$ & $16(53.4 \%)$ & \\
\hline Intratumoral lympho & & & \\
\hline Negative & $2(8.0 \%)$ & $10(33.4 \%)$ & 0.046 \\
\hline Positive & $23(92.0 \%)$ & $20(66.6 \%)$ & \\
\hline Peritumoral lymphoci & & & \\
\hline Negative & $2(8.0 \%)$ & $7(23.4 \%)$ & 0.160 \\
\hline Positive & $23(92.0 \%)$ & $23(76.6 \%)$ & 0.100 \\
\hline Vascular infiltrate & & & \\
\hline Negative & $20(80.0 \%)$ & $28(93.3 \%)$ & 0.226 \\
\hline Positive & $5(20.0 \%)$ & $2(6.7 \%)$ & \\
\hline Perineural infiltrate & & & \\
\hline Negative & $24(96.0 \%)$ & $30(100.0 \%)$ & 0455 \\
\hline Positive & $1(4.0 \%)$ & $0(0.0 \%)$ & 0.453 \\
\hline Tumor markers & & & \\
\hline EGFR & & & \\
\hline Negative & $17(68.0 \%)$ & $24(80.0 \%)$ & 0.363 \\
\hline Positive & $8(32.0 \%)$ & $6(20.0 \%)$ & \\
\hline HER-2 & & & \\
\hline Negative & $25(100 \%)$ & $30(100 \%)$ & Not calculated \\
\hline Positive & 0 & 0 & Not Calcurateda \\
\hline
\end{tabular}


TABle 2: Continued.

\begin{tabular}{|c|c|c|c|}
\hline Variables & + Lymph node metastasis $n(\%)$ & - Lymph node metastasis $n(\%)$ & $p$ \\
\hline \multicolumn{4}{|l|}{ HER-3 } \\
\hline Negative & $1(4.0 \%)$ & $6(20.0 \%)$ & \multirow{2}{*}{0.112} \\
\hline Positive & $24(96.0 \%)$ & $24(80.0 \%)$ & \\
\hline \multicolumn{4}{|l|}{ HER-4 } \\
\hline Negative & $16(65.0 \%)$ & $12(41.4 \%)$ & \multirow{2}{*}{0.083} \\
\hline Positive & $9(35.0 \%)$ & $17(59.6 \%)$ & \\
\hline \multicolumn{4}{|c|}{ Membrane E-cadherin } \\
\hline Negative & $12(54.5 \%)$ & $14(46.7 \%)$ & \multirow{2}{*}{0.779} \\
\hline Positive & $10(45.5 \%)$ & $16(53.3 \%)$ & \\
\hline \multicolumn{4}{|c|}{ Cytoplasm E-cadherin } \\
\hline Negative & $17(73.9 \%)$ & $20(46.6 \%)$ & \multirow{2}{*}{0.764} \\
\hline Positive & $6(26.1 \%)$ & $10(63.4 \%)$ & \\
\hline \multicolumn{4}{|l|}{ Podoplanin } \\
\hline Negative & $13(52.0 \%)$ & $19(63.3 \%)$ & \multirow{2}{*}{0.425} \\
\hline Positive & $12(48.0 \%)$ & $11(36.7 \%)$ & \\
\hline
\end{tabular}

* Only 42 cases.

Cytoplasmic and membranous E-cadherin immunoexpressions were semiquantitatively evaluated as negative if the reaction occurred in up to $50 \%$ of cells and positive if occurred in more than $50 \%$ of cells.

The podoplanin (D2-40) immunoreactivity was semiquantitatively evaluated as described by Padgett et al. [32]: negative: no reactivity or weak reaction independently of the number of cells or moderate/strong reaction in up to $10 \%$ of cells; positive: moderate or intense immunoreaction in more than $10 \%$ of cells.

2.6. Statistics. To analyze the association between clinical variables and lymph node metastasis the chi square, Fisher exact, and $t$-test were used. Specific Cancer Survival (SCS) was also studied, and curves were constructed using the Kaplan-Meier method and compared using the univariate log-rank test. All tests were two sided, and a $P$-value of $\leq .05$ was considered statistically significant. Simultaneous prognostic effect of various factors was determined in a multivariate analysis by use of the Cox proportional-hazards regression model with a covariate of primary interest and adjustment covariates.

\section{Results}

3.1. Demographic, Clinical, and Pathological Descriptive Characteristics. 55 patients with a Mean age of 63 years (Range 30-91) were included in the study.

The median number of mitosis $/ \mathrm{mm}^{2}$ was 3 and median Breslow depth was $8 \mathrm{~mm}$. Tumor characteristics are shown in Table 1.

3.2. Immunohistochemistry. EGFR positivity was $25.5 \%$ in the primary tumor and $41.7 \%$ in the metastases. HER-2 was negative in all samples. HER-3 and HER-4 positivity was $87.3 \%$ and $48.1 \%$ in the primary tumor and $83.3 \%$ and $43.5 \%$ in the metastases, respectively. Membrane Ecadherin positivity was $47.3 \%$ in the primary tumor and $27.3 \%$ in the metastases. Primary tumor cytoplasmic Ecadherin was positive in $30.2 \%$ and $45.5 \%$ in the metastasis. The E-cadherin membrane/cytoplasmic ratio was 1.56 in the primary tumor and 0.60 in the metastases. Podoplanin positivity was $41.8 \%$ in primary tumor and $41.7 \%$ in metastases.

3.3. Risk of Lymph Node Metastasis. Intratumoral lymphocytic infiltrate was the only prognosticator of lymph node metastasis (92\% versus 66.6\%; $p=0.046$ ) (Table 2).

3.4. Survival. The mean and median followup was 9.6 (SD 25.0) and 25.0 months, respectively. At last followup, 19 patients were alive with no evidence of disease $(34.5 \%)$, one was alive with disease $(1.8 \%), 19$ were dead of disease (34.5\%), 9 dead from other causes (16.4\%), and 7 lost to followup (12.7\%). Those lost to followup had a mean and median followup of 24.5 (SD 21.8) and 22.1 months respectively. Only two patients were followed up for less than one year and three patients for less than 22 months. For the 11 patients that presented with lymph node metastasis during the followup, median time to occurrence was 13.08 months.

The overall five years cancer-specific survival (CSS) was $49.7 \%$. For patients with T3 tumors 5 -year CSS was $67.6 \%$, and no patients with T4 tumors were alive at 5 years $(p=0.001)$. Patients with no lymph node metastases had a 5 -year CSS of $63.3 \%$, with no 5 year survivors in patients with lymph node metastases $(p=0.004)$. Gender, race, ambient, anatomic location, location of metastasis, and presence of previous nononcologic lesions did not affect survival (Table 3). There was no difference in 5year CSS between patients with primary tumors with up to 3 mitosis $/ \mathrm{mm}^{2}$ and those with more than 3 mitosis 
TABLE 3: Comparative specific cancer survival rates according to clinical variables in patients with locally advanced cutaneous squamous cell carcinoma of trunk and extremities.

\begin{tabular}{|c|c|c|c|}
\hline Variable & Five years survival $(\%)$ & S.E. & $p$ \\
\hline \multicolumn{4}{|l|}{ Gender } \\
\hline Male & 42.9 & 11.3 & \multirow{2}{*}{0.172} \\
\hline Female & 64.8 & 16.0 & \\
\hline \multicolumn{4}{|l|}{ Race } \\
\hline Caucasian & 50.2 & 10.8 & \multirow{2}{*}{0.443} \\
\hline African & 44.4 & 22.2 & \\
\hline \multicolumn{4}{|l|}{ Ambient } \\
\hline Rural & 71.4 & 17.1 & \multirow{2}{*}{0.598} \\
\hline Urban & 46.0 & 11.1 & \\
\hline \multicolumn{4}{|l|}{ Chronic Sun exposure } \\
\hline Yes & 47.5 & 11.5 & \multirow{2}{*}{0.972} \\
\hline No & 00.0 & 00.0 & \\
\hline \multicolumn{4}{|c|}{ Anatomic location of primary tumor } \\
\hline Lower extremities & 61.0 & 15.5 & \multirow{3}{*}{0.441} \\
\hline Upper extremities & 38.7 & 15.1 & \\
\hline Trunk & 55.6 & 16.6 & \\
\hline \multicolumn{4}{|c|}{ Lymph node metastasis location } \\
\hline Axila & 28.1 & 15.6 & \multirow{2}{*}{0.858} \\
\hline Groin & 00.0 & 00.0 & \\
\hline \multicolumn{4}{|c|}{ Previous non neoplasic lesion } \\
\hline Yes & 39.1 & 18.6 & \multirow{2}{*}{0.221} \\
\hline No & 54.3 & 11.2 & \\
\hline \multicolumn{4}{|l|}{ TNM Classification } \\
\hline $\mathrm{T} 3$ & 67.6 & 10.8 & \multirow{2}{*}{0.001} \\
\hline $\mathrm{T} 4$ & 00.0 & 00.0 & \\
\hline \multicolumn{4}{|l|}{ Lymph node status } \\
\hline No & 63.3 & 10.9 & \multirow{2}{*}{0.004} \\
\hline N1 & 00.0 & 16.8 & \\
\hline \multicolumn{4}{|l|}{ Clinical stage } \\
\hline II & 84.3 & 7.2 & \multirow{2}{*}{$<0.001$} \\
\hline III & 00.0 & 00.0 & \\
\hline
\end{tabular}

(49.7\% versus $47.2 \%, p=0.375)$. No significant difference in 5-year CSS was seen for Breslow depth, peritumoral lymphocytic infiltrate, vascular, and perineural infiltration. The only histological variable that had a significant impact on survival was the tumor grade. Patients with grade I lesions had a 5 -year CSS of $82.2 \%$ compared with $23.8 \%$ in patients with grade II and III tumors $(p=0.010)$. Podoplanin negative patients had a higher 5 years (Figure 1, Table 4 ). In metastatic tumors, HER-4 negativity resulted in a 3year CSS of $66.7 \%$ versus $37.5 \%$ in HER-4 positive patients $(p=0.038)$. Clinical stage and Podoplanin positivity were independent prognostic factors on both univariate and multivariate analysis (Table 5).

\section{Discussion}

4.1. Demographics and Clinical Characteristics. Previous nonskin cancer lesions at the site of disease were seen in
$23 \%$ of patients. Although not associated with lymph node metastasis or poor prognosis, unlike previous reports [6], the presence of noncancerous skin lesions may result in locally advanced disease due to mis- or delayed diagnosis. The $\mathrm{T}$ stage of tumor strongly influenced survival but not incidence of lymph node metastasis. The high number of N1 patients $(25.5 \%)$ has been previously described in an identical setting [10], and may be associated with the two Hospitals involved being tertiary referral oncology centers.

4.2. Pathological Characteristics. Intratumoral lymphocytic infiltration was associated with lymph node metastasis. The inflammatory response may lead to greater tumor antigen exposure in the metastatic lymph node. Tumor thickness (Breslow) had no impact on lymph node metastasis and did not influence survival. Breslow depth may correlate with lymph node metastasis and survival in less advanced tumors, but in this study median tumor thickness was high which 


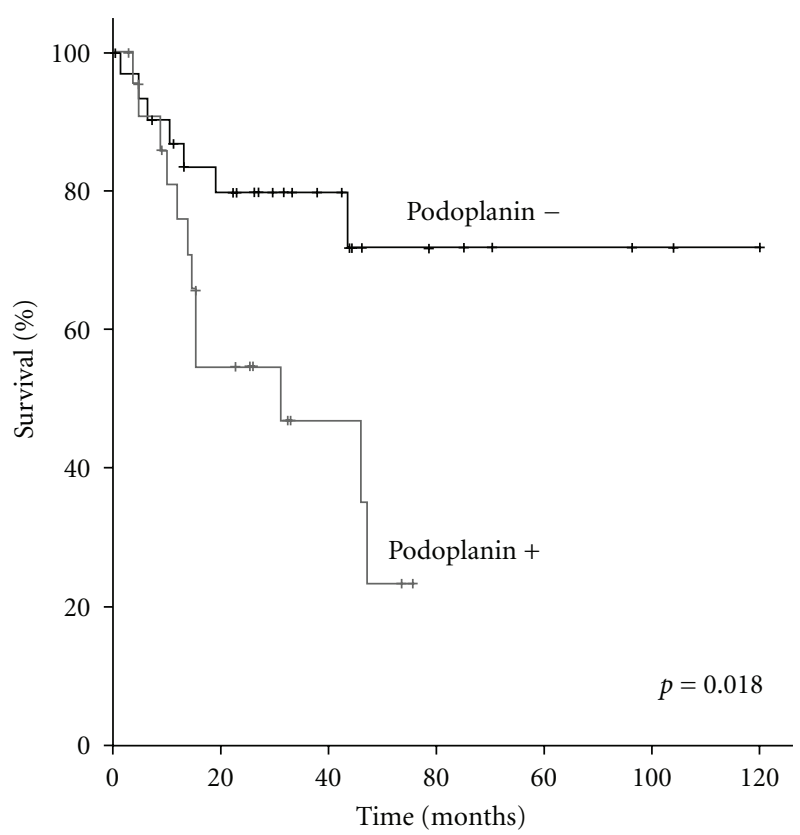

Figure 1

TABle 4: Comparative specific cancer survival rates according to immunohistochemical primary tumor expression in patients with locally advanced cutaneous squamous cell carcinoma of trunk and extremities.

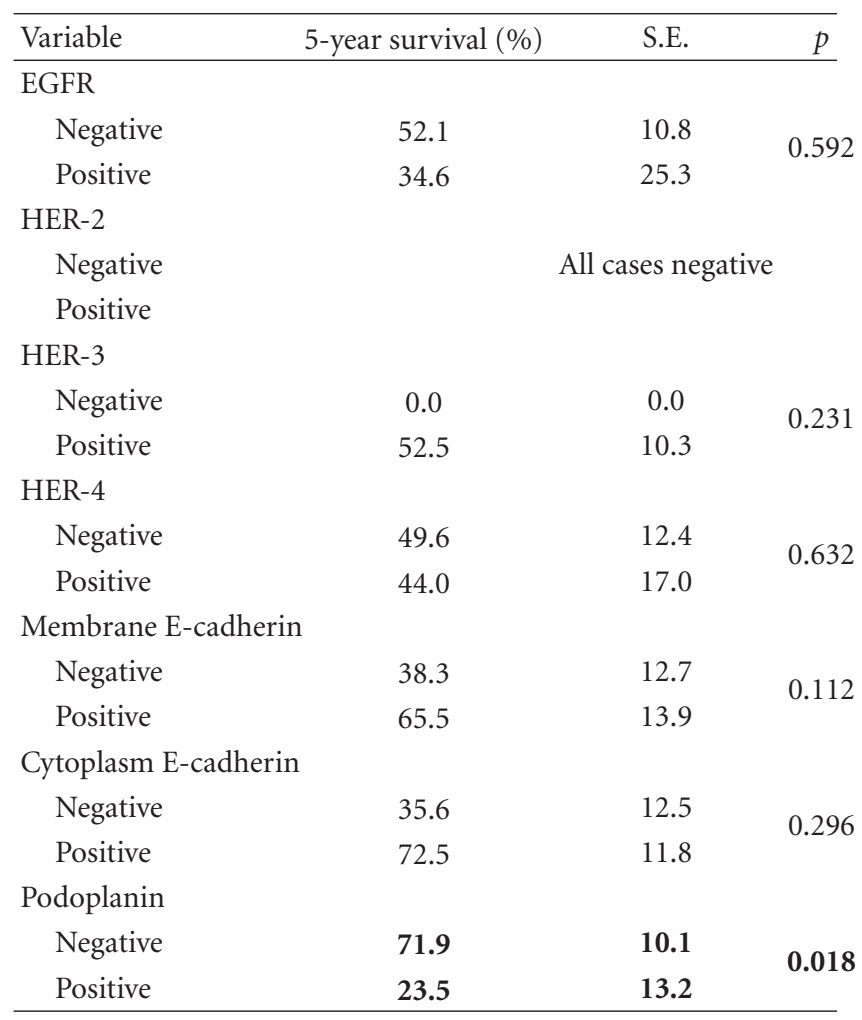

may have weakened any association. Low-grade tumors were associated with prolonged survival confirming the aggressive nature of undifferentiated tumors.
TABle 5: Multivariate Cox regression model for specific cancer survival in patients with locally advanced cutaneous squamous cell carcinoma of trunk and extremities.

\begin{tabular}{lccc}
\hline Variable & HR & $95 \%$ CI & $p$ \\
\hline Clinical stage & & & \\
$\quad$ II & 1 & & 0.003 \\
$\quad$ III & 5.903 & $1.861-18.728$ & \\
Podoplanin & & & \\
$\quad$ Negative & 1 & & 0.050 \\
$\quad$ Positive & 2.839 & $1.011-8.128$ & \\
Age & 1.010 & $0.978-1.044$ & 0.543 \\
Treatment & & & \\
$\quad$ Surgery & 1 & & 0.978 \\
$\quad$ Radiotherapy & 0.981 & $0.254-3.785$ & \\
\hline
\end{tabular}

4.3. Molecular Markers. Unlike squamous cell carcinoma of the head and neck or esophagus, EGFR had no influence on prognosis. It is possible that altered EGFR expression may be associated with local recurrence, which is more frequently life threatening at other sites. HER-2 was negative in all samples and may play little part in CSCC progression as found in squamous cell carcinomas from other sites. High HER-4 expression in lymph node metastases was associated with poor prognosis suggesting a role in progression of CSCC of the trunk and extremities. It is possible that altered HER4 expression occurs late and is present only in metastases. The altered coexpression of the HER family may play a role (i.e., EGFR/HER-4, HER-3/HER-4, and EGFR/HER3/HER-4) but the small number of cases in this study meant this could not be analyzed. E-cadherin expression had no significant association with lymph node metastasis or survival, but the expression ratio between membrane and cytoplasm was lower in the metastasis, suggesting accumulation with a loss of function. This may be due to a mutated E-cadherin resulting in cytoplasmic accumulation with a loss of cell adhesion and disease progression $[33,34]$. Some studies have looked at the membrane expression of Ecadherin in cutaneous squamous cell carcinoma compared with normal skin, local tumors, and metastasis, suggesting a progressive loss of expression [35-37].

Podoplanin expression was not associated with the presence of lymph node metastasis, but was a prognosticator of reduced survival indicating a locally aggressive tumor, with survival impact. Altered expression of podoplanin is associated with mesothelioma, squamous cell carcinoma of oral mucosa, and germ cell tumors, suggesting that podoplanin may influence invasive and proliferative activity [38-40]. As CSCC metastases occur preferentially via lymphatic vessels, podoplanin expression may be associated with disease progression. Hyperexpression has been related to undifferentiated skin tumors, but its impact on prognosis and metastasis has not been established [41]. Podoplanin is a possible target for development of novel therapies, and its expression has to be studied in other settings to completely understand its role in cancer development and progression. 
Patients with advanced CSCC of trunk and extremities with poor prognostic factors such as undifferentiated, T4, N1 tumors, high podoplanin expression in the primary tumor, or high HER-4 expression in the lymph node metastasis may be candidates for new more aggressive modalities of treatment. Further studies using these molecular markers are needed to help refine treatment of CSCC of the trunk and extremities.

\section{Conflict of Interests}

The authors declare that there is no conflict of interests.

\section{Acknowledgment}

This work was funded by the Research and Education Institute, Barretos Cancer Hospital, Barretos, Brazil.

\section{References}

[1] R. Marks, "An overview of skin cancers. Incidence and causation," Cancer, vol. 75, no. 2, pp. 607-612, 1994.

[2] M. Verschoore, "The epidemiology of skin cancer," British Journal of Dermatology, vol. 146, no. 61, supplement, pp. 16, 2002.

[3] "Cancer incidence in five continents," IARC Scientific Publications, vol. 9, no. 160, pp. 1-837, 2008.

[4] C. J. Mettlin, "Guest editorial: skin cancer and ozone depletion: the case for global action," Journal of Surgical Oncology, vol. 77, no. 2, pp. 76-78, 2001.

[5] C. Garbe and U. Leiter, "Epidemiology of melanoma and nonmelanoma skin cancer-the role of sunlight," Advances in Experimental Medicine and Biology, vol. 624, pp. 89-103, 2008.

[6] V. de Lima Vazquez, T. Sachetto, N. M. Perpetuo, and A. L. Carvalho, "Prognostic factors for lymph node metastasis from advanced squamous cell carcinoma of the skin of the trunk and extremities," World Journal of Surgical Oncology, vol. 6, article 73, 2008.

[7] M. G. Joseph, W. P. Zulueta, and P. J. Kennedy, "Squamous cell carcinoma of the skin of the trunk and limbs: the incidence of metastases and their outcome," Australian and New Zealand Journal of Surgery, vol. 62, no. 9, pp. 697-701, 1992.

[8] D. Czarnecki, M. Staples, A. Mar, G. Giles, and C. Meehan, "Metastases from squamous cell carcinoma of the skin in southern Australia," Dermatology, vol. 189, no. 1, pp. 52-54, 1994.

[9] L. Rosenblatt and R. Marks, "Deaths due to squamous cell carcinoma in Australia: is there a case for a public health intervention?" Australasian Journal of Dermatology, vol. 37, no. 1, pp. 26-29, 1996.

[10] J. H. North, J. E. Spellman, D. Driscoll, A. Velez, W. G. Kraybill, and N. J. Petrelli, "Advanced cutaneous squamous cell carcinoma of the trunk and extremity: analysis of prognostic factors," Journal of Surgical Oncology, vol. 64, no. 3, pp. 212217, 1997.

[11] J. T. Mullen, L. Feng, Y. Xing et al., "Invasive squamous cell carcinoma of the skin: defining a high-risk group," Annals of Surgical Oncology, vol. 13, no. 7, pp. 902-909, 2006.

[12] B. S. Cherpelis, C. Marcusen, and P. G. Lang, "Prognostic factors for metastasis in squamous cell carcinoma of the skin," Dermatologic Surgery, vol. 28, no. 3, pp. 268-273, 2002.
[13] F. C. Ames and R. C. Hickey, "Metastasis from squamous cell skin cancer of the extremities," Southern Medical Journal, vol. 75, no. 8, pp. 920-932, 1982.

[14] D. H. Kraus, J. F. Carew, and L. B. Horrison, "Regional lymph node metastasis from cutaneous squamous cell carcinoma," Archives of Otolaryngology, vol. 124, no. 5, pp. 582-587, 1998.

[15] B. A. Moore, R. S. Weber, V. Prieto et al., "Lymph node metastases from cutaneous squamous cell carcinoma of the head and neck," Laryngoscope, vol. 115, no. 9, pp. 1561-1567, 2005.

[16] B. Linggi and G. Carpenter, "ErbB receptors: new insights on mechanisms and biology," Trends in Cell Biology, vol. 16, no. 12, pp. 649-656, 2006.

[17] D. J. Slamon, G. M. Clark, and S. G. Wong, "Human breast cancer: correlation of relapse and survival with amplification of the HER-2/neu oncogene," Science, vol. 235, no. 4785, pp. 177-182, 1987.

[18] S. Xu, J. Kitayama, H. Yamashita, D. Souma, and H. Nagawa, "Nuclear translocation of HER-4/c-erbB-4 is significantly correlated with prognosis of esophageal squamous cell carcinoma," Journal of Surgical Oncology, vol. 97, no. 1, pp. 44-50, 2008.

[19] S. M. Wiseman, N. Makretsov, T. O. Nielsen et al., "Coexpression of the type 1 growth factor receptor family members HER-1, HER-2, and HER-3 has a synergistic negative prognostic effect on breast carcinoma survival," Cancer, vol. 103, no. 9, pp. 1770-1777, 2005.

[20] R. Roskoski Jr., “The ErbB/HER receptor protein-tyrosine kinases and cancer," Biochemical and Biophysical Research Communications, vol. 319, no. 1, pp. 1-11, 2004.

[21] G. Krähn, U. Leiter, P. Kaskel et al., "Coexpression patterns of EGFR, HER2, HER3 and HER4 in non-melanoma skin cancer," European Journal of Cancer, vol. 37, no. 2, pp. 251259, 2001.

[22] N. U. Ahmed, M. Ueda, and M. Ichihashi, "Increased level of c-erbB-2/neu/HER-2 protein in cutaneous squamous cell carcinoma," British Journal of Dermatology, vol. 136, no. 6, pp. 908-912, 1997.

[23] G. B. Fogarty, N. M. Conus, J. Chu, and G. McArthur, "Characterization of the expression and activation of the epidermal growth factor receptor in squamous cell carcinoma of the skin," British Journal of Dermatology, vol. 156, no. 1, pp. 92-98, 2007.

[24] E. Maubec, P. Duvillard, V. Velasco, B. Crickx, and M. F. Avril, "Immunohistochemical analysis of EGFR and HER-2 in patients with metastatic squamous cell carcinoma of the skin," Anticancer Research, vol. 25, no. 2, pp. 1205-1210, 2005.

[25] B. P. L. Wijnhoven, W. N. M. Dinjens, and M. Pignatelli, "E-cadherin-catenin cell-cell adhesion complex and human cancer," British Journal of Surgery, vol. 87, no. 8, pp. 992-1005, 2000.

[26] F. Nollet, G. Berx, and F. Van Roy, "The role of the E-cadherin/catenin adhesion complex in the development and progression of cancer," Molecular Cell Biology Research Communications, vol. 2, no. 2, pp. 77-85, 1999.

[27] V. Schacht, M. I. Ramirez, Y. K. Hong et al., "T1 $\alpha /$ podoplanin deficiency disrupts normal lymphatic vasculature formation and causes lymphedema," EMBO Journal, vol. 22, no. 14, pp. 3546-3556, 2003.

[28] T. Brenn and P. Mckee, "Tumor of the surface epithelium: squamous cell carcinoma," in Pathology of the Skin with Clinical Correlations, P. C. Mckee and E. Granter Sr., Eds., pp. 1199-1209, Elsevier Mosby, London, UK, 2005. 
[29] S. M. Hsu and L. Raine, "Protein A, avidin, and biotin in immunohistochemistry," Journal of Histochemistry and Cytochemistry, vol. 29, no. 11, pp. 1349-1353, 1981.

[30] D. J. Lager, D. D. Slagel, and P. L. Palechek, “The expression of epidermal growth factor receptor and transforming growth factor alpha in renal cell carcinoma," Modern Pathology, vol. 7, no. 5, pp. 544-548, 1994.

[31] L. Shen, Y. Shui, X. Wang et al., "EGFR and HER2 expression in primary cervical cancers and corresponding lymph node metastases: implications for targeted radiotherapy," BMC Cancer, vol. 8, article 232, 2008.

[32] D. M. Padgett, H. P. Cathro, M. R. Wick, and S. E. Mills, "Podoplanin is a better immunohistochemical marker for sarcomatoid mesothelioma than calretinin," American Journal of Surgical Pathology, vol. 32, no. 1, pp. 123-127, 2008.

[33] B. Nawrocki, M. Polette, J. Van Hengel, J. M. Tournier, F. Van Roy, and P. Birembaut, "Cytoplasmic redistribution of E-cadherin-catenin adhesion complex is associated with down-regulated tyrosine phosphorylation of E-cadherin in human bronchopulmonary carcinomas," American Journal of Pathology, vol. 153, no. 5, pp. 1521-1530, 1998.

[34] R. M. Bremnes, R. Veve, E. Gabrielson et al., "Highthroughput tissue microarray analysis used to evaluate biology and prognostic significance of the E-cadherin pathway in nonsmall-cell lung cancer," Journal of Clinical Oncology, vol. 20, no. 10, pp. 2417-2428, 2002.

[35] A. Lyakhovitsky, A. Barzilai, M. Fogel, H. Trau, and M. Huszar, "Expression of E-cadherin and beta-catenin in cutaneous squamous cell carcinoma and its precursors," American Journal of Dermatopathology, vol. 26, no. 5, pp. 372-378, 2004.

[36] S. Koseki, T. Aoki, S. Ansai, Y. Hozumi, Y. Mitsuhashi, and S. Kondo, "An immunohistochemical study of E-cadherin expression in human squamous cell carcinoma of the skin: relationship between decreased expression of E- cadherin in the primary lesion and regional lymph node metastasis," Journal of Dermatology, vol. 26, no. 7, pp. 416-422, 1999.

[37] H. Wu, R. Lotan, D. Menter, S. M. Lippman, and X. C. Xu, "Expression of e-cadherin is associated with squamous differentiation in squamous cell carcinomas," Anticancer Research, vol. 20, no. 3 A, pp. 1385-1390, 2000.

[38] P. A. Kyzas, S. Geleff, A. Batistatou, N. J. Agnantis, and D. Stefanou, "Evidence for lymphangiogenesis and its prognostic implications in head and neck squamous cell carcinoma," Journal of Pathology, vol. 206, no. 2, pp. 170-177, 2005.

[39] N. G. Ordóñez, "D2-40 and podoplanin are highly specific and sensitive immunohistochemical markers of epithelioid malignant mesothelioma," Human Pathology, vol. 36, no. 4, pp. 372-380, 2005.

[40] H. Yu, G. S. Pinkus, and J. L. Hornick, "Diffuse membranous immunoreactivity for podoplanin (D2-40) distinguishes primary and metastatic seminomas from other germ cell tumors and metastatic neoplasms," American Journal of Clinical Pathology, vol. 128, no. 5, pp. 767-775, 2007.

[41] H. M. Yang, E. Cabral, S. S. Dadras, and D. S. Cassarino, "Immunohistochemical expression of D2-40 in benign and malignant sebaceous tumors and comparison to basal and squamous cell carcinomas," American Journal of Dermatopathology, vol. 30, no. 6, pp. 549-554, 2008. 


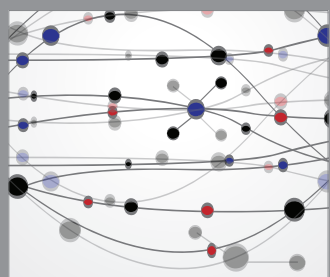

The Scientific World Journal
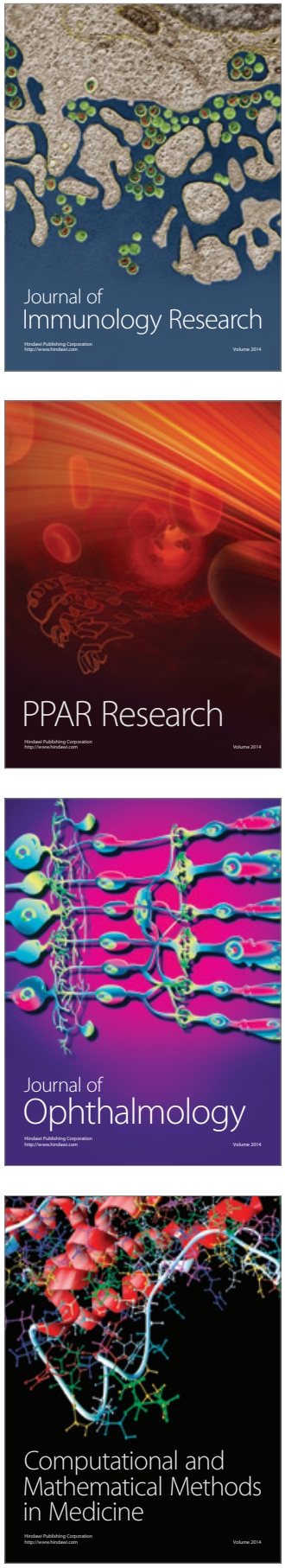

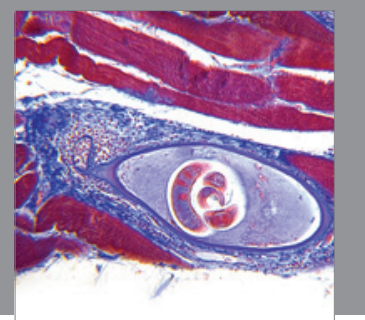

Gastroenterology

Research and Practice
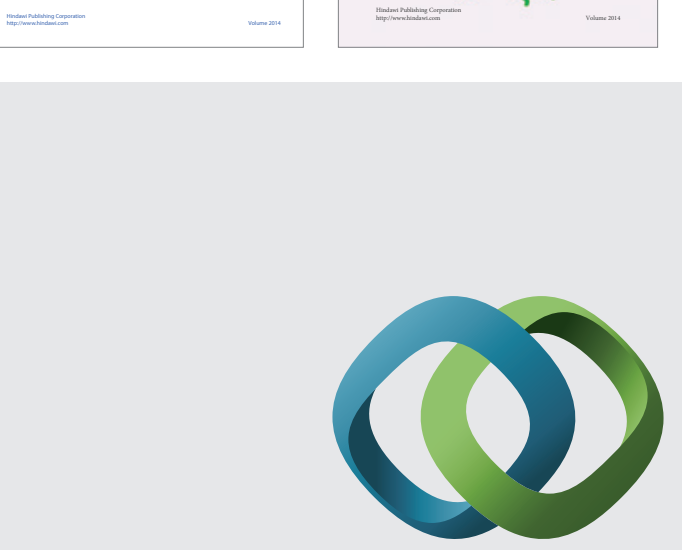

\section{Hindawi}

Submit your manuscripts at

http://www.hindawi.com
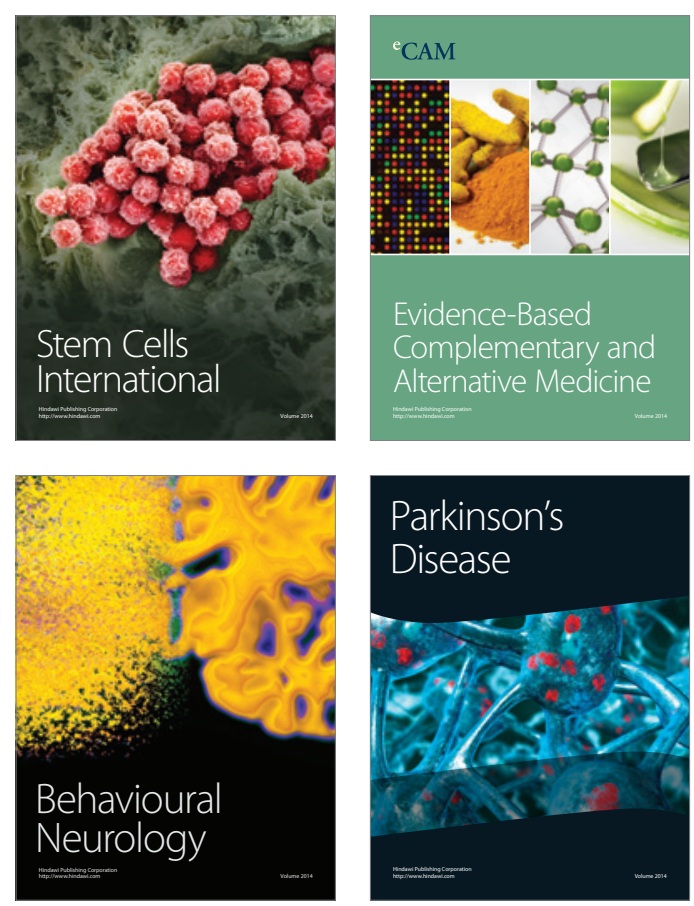

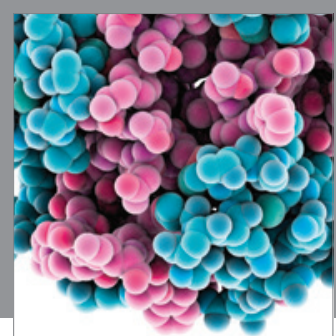

Journal of
Diabetes Research

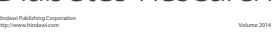

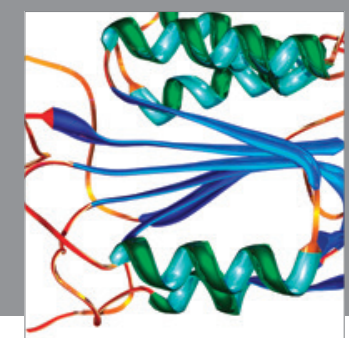

Disease Markers
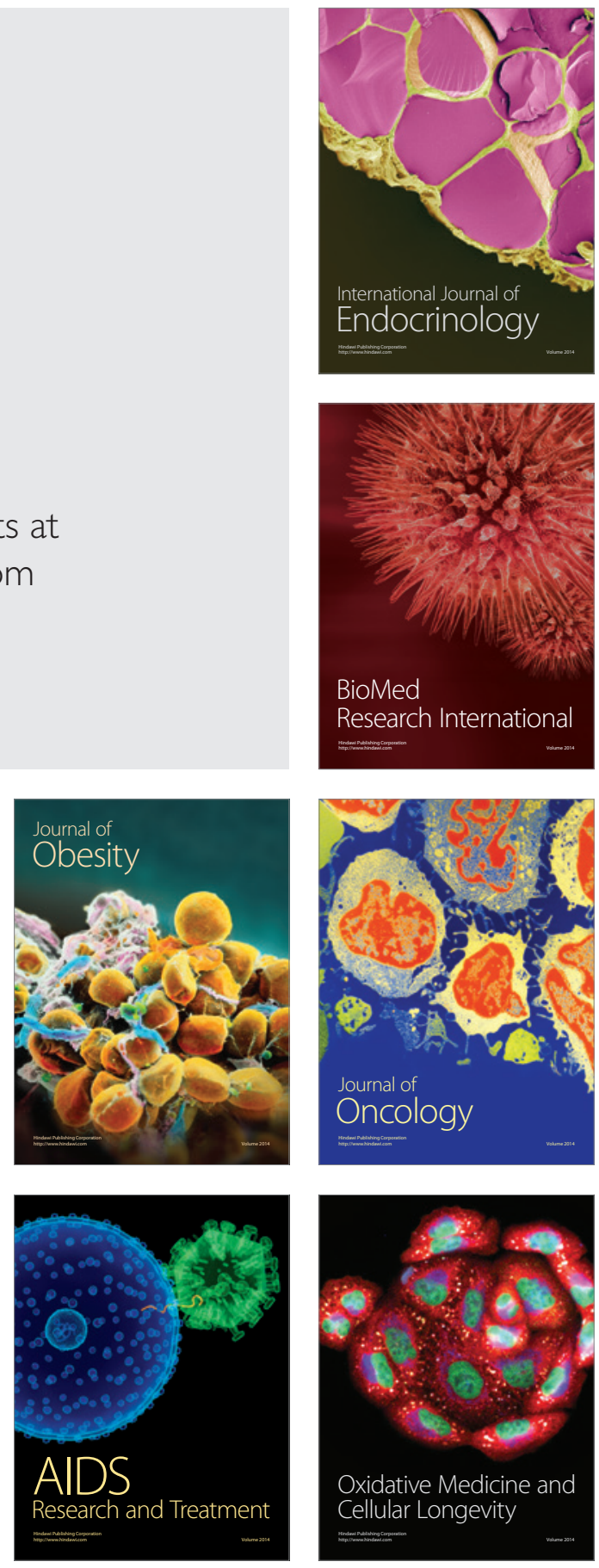